\section{- Chinese Pharmacists Propose Patient Consent for Unlabeled Use of Medications}

In the United States, "unlabeled uses" are defined as drug uses that not included in the indications or dosage regimens listed in the U.S. Food and Drug Administration (FDA)-approved labeling. Unlabeled uses include the uses of a drug product in (1) doses, (2) patient populations, (3) indications, or (4) routes of administration that are not reflected in FDA-approved product labeling. ${ }^{.}$In April 1982, the FDA published a statement entitled "Use of Approved Drugs for Unlabeled Indications" to address the issues of appropriateness and legality of prescribing approved drugs for uses not included in FDA-approved labeling. The FDA specified that unlabeled use of medications may be appropriate and rational in certain circumstances. ${ }^{2}$

In China, however, there are no regulations to date from the China State Food and Drug Administration (SFDA) on the use of medications for unlabeled uses. Therefore, the legal issues surrounding unlabeled uses of medications arouse controversy in the whole country. Advocates of permitting unlabeled use believe that the freedom and responsibility to make drug therapy decisions that are consistent with patient needs is a fundamental precept and it is a professional duty of physicians and pharmacists that should not be limited by language in SFDA-approved product labeling. Opponents of off-label use emphasize that drug labeling approved by SFDA is the legal document for medical liability management and insist that physicians must prescribe drugs according to the product labeling; to do otherwise should be regarded as rule violation.

Currently, in many cases the indications and usage in product labeling in China lag behind medical practice and the literature. Meanwhile the use of medications for unlabeled uses has been common practice in hospitals in China for a long time. ${ }^{3}$ Physicians often prescribe a medication for a purpose other than that for which it has been specifically designed and approved by SFDA.

In order to minimize the risk for physicians and pharmacists and to protect patients, there is a need to standardize the use of medications for unlabeled uses. Accordingly, 20 senior chief pharmacists from 17 hospitals in Guangdong province, created a consensus statement on the use of medications for unlabeled uses in March, 2010. ${ }^{4}$ The consensus statement includes 5 elements:

(1) The use of the drug for unlabeled use means that there is no effective drug that can be used according to present labeling to treat the patient's condition. The drug for unlabeled use is irreplaceable for treatment of the patient.
Before prescribing the drug for unlabeled use, adverse drug reactions, contraindications, and warnings should be checked carefully to ensure that benefit outweighs risk as used in the patient.

(2) The aim of unlabeled use is for the patient's interest/benefit and not for clinical trial or clinical research. This is medical staff's professional entitlement.

(3) The medication for unlabeled use should be based on the reasonable medical practice evidence, including sufficient reports in medical literature, long-term practice, etc.

(4) An application for unlabeled uses should be submitted and approved by the hospital's pharmacotherapy committee and hospital ethics committee. However, an unlabeled use may be exempted from application for approval in an emergency situation.

(5) Oral or written informed consent should be obtained from the patient or from the guardian of the patient who would be administered the drug for unlabeled use.

\section{Zhihua Zheng, PharmD}

Chief Pharmacist

Deputy Secretary-General for Guangdong Province

Pharmaceutical Association, Guangzhou 510080, China

snownotrace@gmail.com

\section{Feng $X u, P h D$}

Professor of Clinical Pharmacology

Second Medical College \& Zhujiang Hospital

Southern Medical University, Guangzhou 510280, China

andrewfxu1998@gmail.com

\section{DISCLOSURES}

The authors report no potential bias or conflicts of interest related to this letter.

\section{REFERENCES}

1. No authors listed. ASHP statement on the use of medications for unlabeled uses. Am J Hosp Pharm. 1992;49(8):2006-08. Available at: http://www. ashp.org/DocLibrary/BestPractices/FormStUnlabeled.aspx. Accessed August 29, 2010.

2. No authors listed. Use of approved drugs for unlabeled indications. FDA Drug Bull. 1982;12(1):4-5.

3. Xu F. Unlabeled Uses for Common Diseases. 2nd edition. Beijing: China Medicinal Press; 2005.

4. Guangdong Province Pharmaceutical Association. Consensus on the use of medications for unlabeled uses. Pharm Today, 2010;20(4):1-3. Available at: http://www.jinriyaoxue.com/Magazine/Show.aspx?Id=1006. Accessed August 29, 2010. 